\title{
Causas infrecuentes de proptosis aguda dolorosa. Reporte de
} casos

\section{Infrequent causes of acute painful proptosis. Case series}

\author{
Yanelis Maimó-Gallego ${ }^{1 *}$, Tamara Juvier-Riesgo ${ }^{1}$, Noemy Bory-Vargas ${ }^{1}$ y Adnolys Reyes-Berazaín ${ }^{2}$ \\ ${ }^{1}$ Departamento de Neuro-Oftalmología; ${ }^{2}$ Departamento de Neuro-Imagenología. Instituto de Neurología y Neurocirugía Prof. Dr. Rafael Estrada, La \\ Habana, Cuba
}

\section{Resumen}

Introducción: Los trastornos del drenaje venoso orbitario incluyen proptosis de inicio brusco, edema de los párpados, dilatación de los vasos venosos epiesclerales, hipertensión ocular y oftalmoplejía. La resonancia magnética es el mejor método para identificar la causa. Casos clínicos: Se reportan dos casos inusuales, en pacientes de 54 y 56 años de edad. Se presentaron con proptosis dolorosa aguda. Uno de ellos desarrolló edema de párpados, quemosis, dilatación y tortuosidad de los vasos venosos epiesclerales, así como hipertensión ocular. En este caso se detectó una trombosis de la vena oftálmica superior izquierda. El segundo paciente experimentó síntomas clínicos similares, pero se diagnosticó una metástasis en el seno cavernoso izquierdo. Estas afecciones son diferentes variantes de trastornos del drenaje venoso orbitario con un comportamiento clínico similar, aunque de diferentes causas. Estos diagnósticos deben considerarse en todo paciente con proptosis aguda dolorosa. Se han reportado pocos casos en la literatura.

Palabras clave: Proptosis. Dolor ocular. Trombosis de vena oftálmica superior. Síndrome del seno cavernoso. Oftalmoplejía dolorosa. Metástasis.

\section{Abstract}

Introduction: Orbital venous drainage disorders include a sudden onset of proptosis, eyelid edema, episcleral vascular engorgement and tortuosity, elevated intraocular pressure, and ophthalmoplegia. Magnetic resonance imaging is the best method to identify the cause. Case reports: We report two unusual cases in patients of 54 and 56 years of age. They presented with acute painful proptosis. One of them developed eyelid edema, chemosis, episcleral vascular engorgement and tortuosity, as well as ocular hypertension. Thrombosis of the left superior ophthalmic vein was detected in this case. The second patient experienced similar clinical symptoms but was diagnosed with a left cavernous sinus metastasis. These conditions are different variants of orbital venous drainage disorders with similar clinical characteristics but different causes. These diagnoses should be considered in any patient with acute painful proptosis. Few cases have been reported in the literature.

Key words: Proptosis. Ocular pain. Superior ophthalmic vein thrombosis. Cavernous sinus syndrome. Painful ophthalmoplegia. Metastasis.

\section{Correspondencia:}

*Yanelis Maimó-Gallego 


\section{Introducción}

La vena oftálmica superior se origina en el ángulo superior interno de la cavidad orbitaria y sigue su curso con la arteria orbital superior para drenar en el seno cavernoso. Cualquier alteración que afecte el drenaje venoso de la órbita puede producir proptosis, edema palpebral, dilatación de vasos venosos epiesclerales, hipertensión ocular y limitación de la motilidad ocular ${ }^{1}$.

Las alteraciones vasculares (fístula carotidocavernosa, sangrado de malformaciones vasculares) e inflamatorias (pseudotumor orbitario y síndrome de Tolosa-Hunt) con frecuencia producen este cuadro clínico de forma aguda, por lo que son estos los diagnósticos etiológicos que se plantean en primer lugar ante un caso de proptosis aguda dolorosa asociada a otros signos $^{2-4}$. La resonancia magnética (RM) constituye el mejor método identificado para el diagnóstico de estas afecciones ${ }^{5}$.

La trombosis de la vena oftálmica superior aislada y la metástasis a nivel del seno cavernoso no se han reportado con frecuencia, por lo que presentamos dos casos con dichos diagnósticos.

\section{Caso clínico 1}

Mujer de 54 años que acudió a consulta por enrojecimiento, dolor e inflamación en el ojo izquierdo, de aparición brusca 1 mes antes. Se recoge el antecedente de neoplasia ginecológica, que fue tratada con radioterapia y quimioterapia, 3 años antes. Es fumadora de 1 cajetilla diaria de cigarros.

- Psicofísica visual: agudeza visual, visión al color y test de sensibilidad al contraste normal en ambos ojos.

- Examen físico ocular: ojo derecho normal. Ojo izquierdo: edema palpebral, quemosis, tortuosidad y dilatación de vasos venosos epiesclerales (Fig. 1 A).

- Segmento anterior: normal en ambos ojos.

- Presión ocular: $17 \mathrm{mmHg}$ en el ojo derecho y 32 $\mathrm{mmHg}$ en el ojo izquierdo.

- Presión ocular en supraducción: 18 mmHg en el ojo derecho y $37 \mathrm{mmHg}$ en el ojo izquierdo.

- Alineación ocular en posición primaria de la mirada: ortotropía en ambos ojos.

- Cover test: no movimientos de refijación en ambos ojos.

- Movimientos oculares: ducciones sin limitación en ambos ojos.
- Exoftalmometría: $4 \mathrm{~mm}$ de proptosis del ojo izquierdo.

- Fondo de ojo: normal en el ojo derecho y con dilatación de vasos venosos en el ojo izquierdo.

Se indica RM de cráneo y órbita (Fig. 1 B), en la que se encuentra la vena oftálmica superior izquierda aumentada de tamaño e hiperintensa en flair y T2, músculos extraoculares izquierdos engrosados y proptosis de ese lado; hallazgos que sugieren el diagnóstico de trombosis de la vena oftálmica izquierda superior.

\section{Caso clínico 2}

Varón de 56 años que pesentó de forma brusca, después de un cuadro de estrés, caída del párpado superior izquierdo, así como inflamación y dolor ocular en el mismo ojo. Al elevarse el párpado notaba diplopía. Se recoge el antecedente de haber sido operado de un hipernefroma hace 6 años.

- Psicofísica visual: agudeza visual, visión al color y test de sensibilidad al contraste normal en ambos ojos.

- Examen físico ocular: ojo derecho normal. Ojo izquierdo: edema y ptosis palpebral, quemosis, tortuosidad y dilatación de vasos venosos epiesclerales (Fig. 2 A y B).

- Segmento anterior: normal en ambos ojos.

- Medios: transparentes en ambos ojos.

- Presión ocular: $11 \mathrm{mmHg}$ en el ojo derecho y 36 $\mathrm{mmHg}$ en el ojo izquierdo.

- Exoftalmometría: $7 \mathrm{~mm}$ de proptosis en el ojo izquierdo.

- Alineación en posición primaria de la mirada: exotropía $\left(20^{\circ}\right)$ con hipotropía en el ojo izquierdo.

- Motilidad ocular: en ojo izquierdo limitación de la motilidad de todos los músculos extraoculares (Fig. 2 B).

- Pupila: en el ojo izquierdo en posición media, con defecto pupilar eferente.

- Fondo de ojo: en el ojo izquierdo vasos venosos dilatados y tortuosos, sin otras alteraciones.

Se indica RM (Fig. 3) de cráneo y órbita, en la que se encuentra una lesión que ocupa el seno cavernoso izquierdo, que repercute en la órbita homolateral, produciendo congestión de esta, evidente por la proptosis, engrosamiento de músculos orbitarios, alteración de la intensidad de señales de la grasa orbitaria y engrosamiento de la vena oftálmica superior homolateral. Se observa, además, en la región parietooccipital derecha, una lesión nodular de 1,6 × 1,5 cm rodeada por edema, con efecto de masa sobre el parénquima 


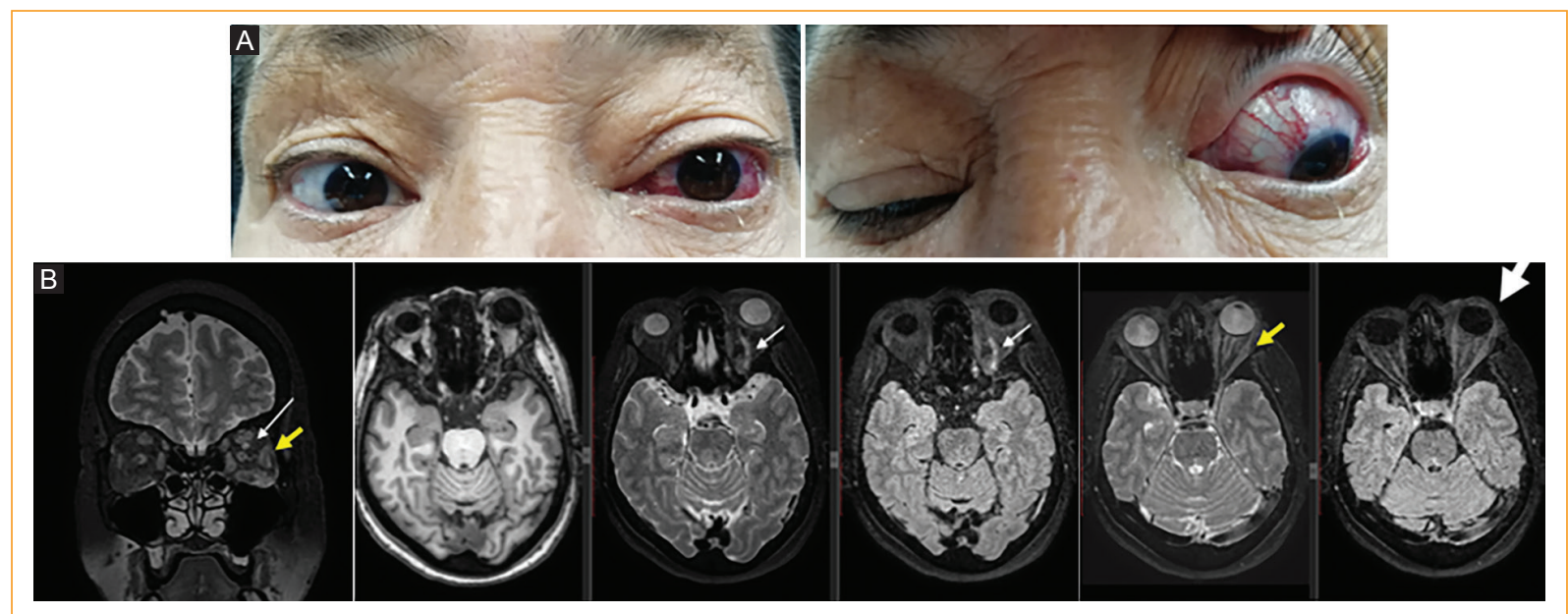

Figura 1. A: edema palpebral. Quemosis, tortuosidad y dilatación de vasos venosos epiesclerales. B: resonancia magnética 3T de cráneo y órbita que muestra la vena oftálmica izquierda aumentada de tamaño e hiperintensa en flair y T2 (flecha fina y larga), músculos extraoculares izquierdos engrosados (flecha corta) y proptosis izquierda (flecha gruesa).
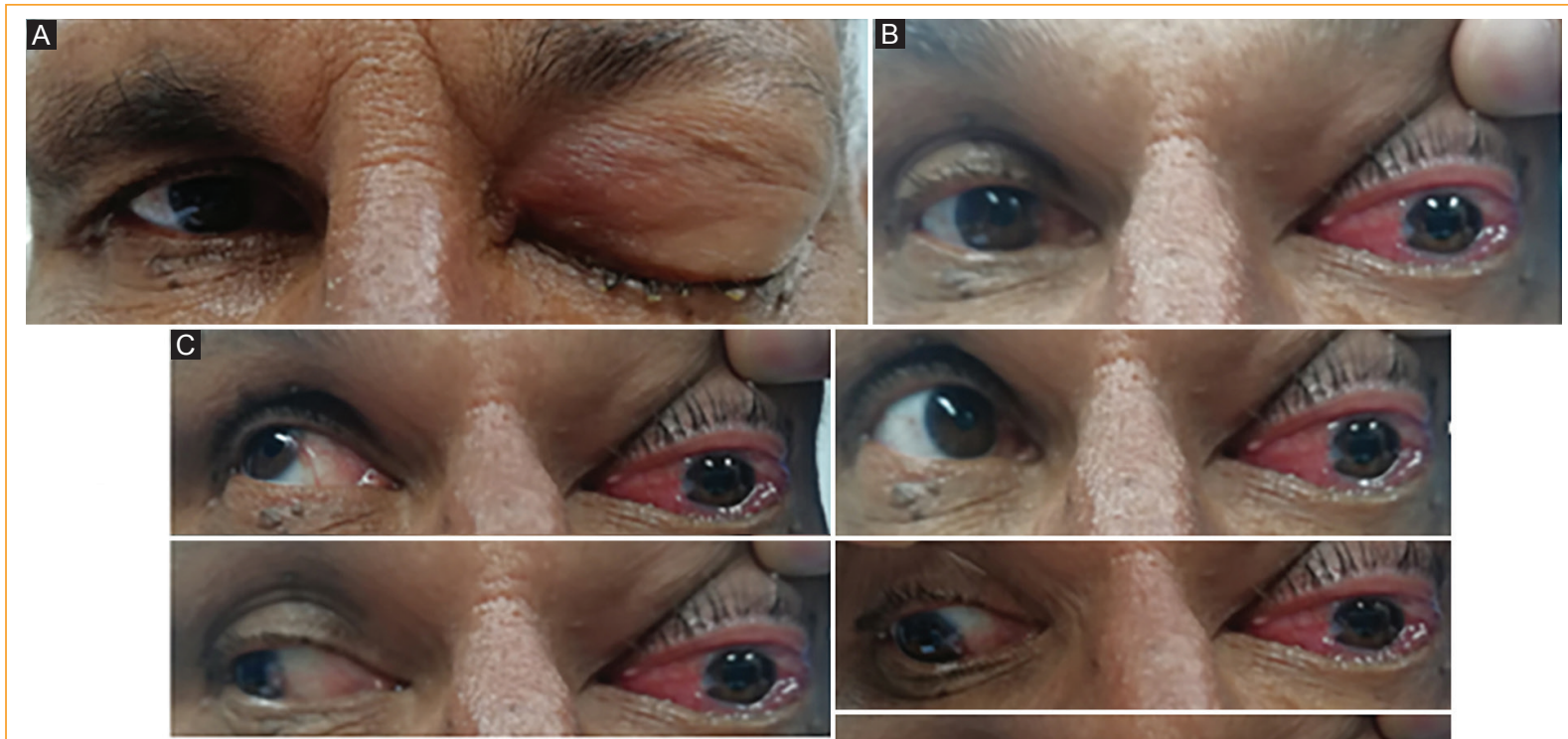

Diferentes posiciones de la mirada

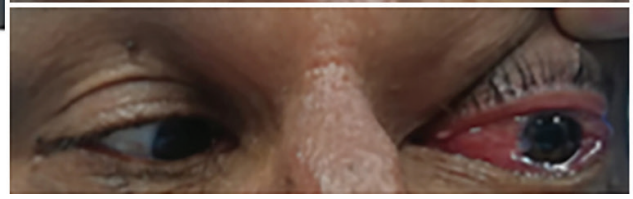

Figura 2. A: edema y ptosis palpebral en el ojo izquierdo. B: quemosis, tortuosidad y dilatación de vasos venosos epiesclerales. C: oftalmoplejía.

vecino y el sistema ventricular adyacente, de naturaleza secundaria.

Dados los antecedentes del paciente y las características imagenológicas, se plantea enfermedad oncoproliferativa y como causa del cuadro clínico la metástasis en el seno cavernoso.

\section{Discusión}

Cuando se evalúa un paciente con proptosis aguda dolorosa se piensa en enfermedades inflamatorias o vasculares de la órbita o del seno cavernoso, cuyas causas más frecuentes son la fístula carotidocavernosa, la 


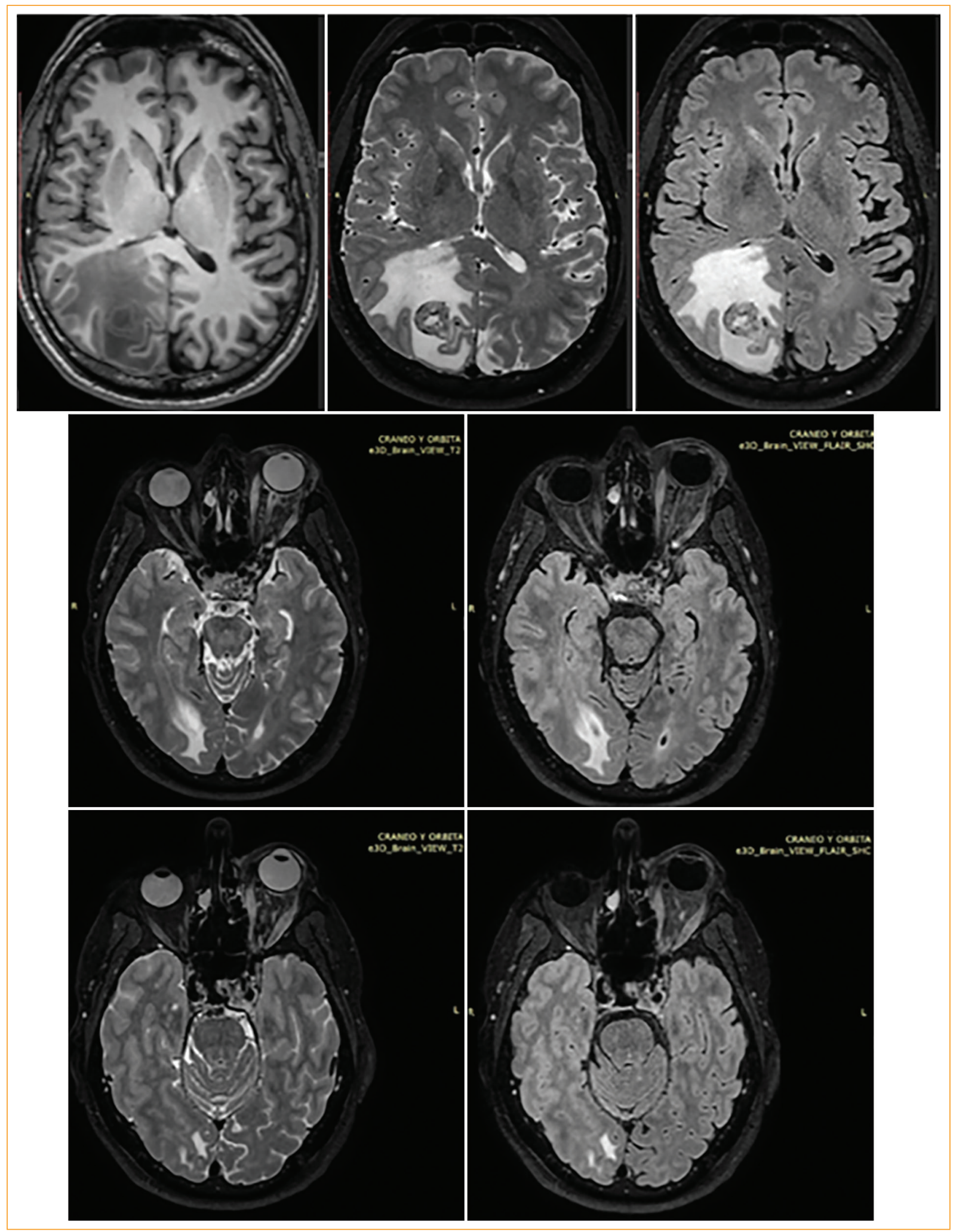

Figura 3. Resonancia magnética 3T axial, de cráneo y órbita, que muestra una lesión nodular en la región parietooccipital derecha rodeada por edema, con efecto de masa sobre el parénquima vecino y el sistema ventricular adyacente. Lesión ocupativa del seno cavernoso izquierdo con repercusión en la órbita homolateral (proptosis ocular, engrosamiento de músculos orbitarios, alteración de la intensidad de señales de la grasa orbitaria y engrosamiento de la vena oftálmica superior). 
trombosis venosa de seno cavernoso, la celulitis orbitaria y el pseudotumor orbitario; sin embargo, cualquier situación que produzca congestión en el drenaje venoso de la órbita puede producir el cuadro. Otras afecciones, como la trombosis de la vena oftálmica superior y las metástasis del seno cavernoso, son menos frecuentes, pero no improbables. El diagnóstico diferencial mediante la clínica resulta difícil, sobre todo cuando no hay antecedente de trauma ni de infección ${ }^{3,6-8}$, y en estos casos la RM es la herramienta más sensible y específica ${ }^{8-11}$.

La primera paciente que aquí reportamos tuvo un cuadro que sugería patología orbitaria, con una presentación aguda, presuntamente de naturaleza inflamatoria o vascular; sin embargo, los síntomas y los signos fueron insuficientes para presumir la causa, por lo que la RM tuvo un papel fundamental en el diagnóstico etiológico.

La trombosis de la vena oftálmica superior aislada es una patología orbital infrecuente que se puede presentar con proptosis de inicio rápido, inyección conjuntival y trastornos visuales. Las pruebas de imagen, como la RM, son más sensibles y pueden detectar una vena oftálmica engrosada y dilatada. La trombosis de la vena oftálmica superior aparece con más frecuencia debido a inflamaciones o infecciones orbitales, a compresión o a infiltración de tumores, así como a consecuencia de una fístula carotidocavernosa ${ }^{12-17}$.

Además de las enfermedades orbitales y del seno cavernoso, hay factores de riesgo sistémicos, como estados de hipercoagulabilidad o amiloidosis, así como enfermedades inflamatorias y malignas que pueden predisponer a la trombosis de la vena oftálmica superior $^{12,18-21}$. Otros elementos, como los que componen la tríada de Virchow (estasis vascular, daño endotelial e hipercoagulabilidad), desempeñan un papel importante en la fisiopatología ${ }^{19}$.

Aunque no existe en la literatura revisada gran número de reportes de casos con trombosis aisladas, algunos autores presentan pacientes con igual sintomatología que la nuestra, sin encontrar una causa local específica que la produzca ${ }^{17,21,22}$.

El caso presentado de trombosis de la vena oftálmica tiene como único antecedente el diagnóstico de una neoplasia ginecológica y el uso de quimioterapia. Habib y Lessard ${ }^{18}$ reportan un caso de trombosis de la vena oftálmica superior aislada asociada a cáncer de pulmón.

El segundo paciente presentó un cuadro de proptosis aguda dolorosa que se acompañó clínicamente de un síndrome de seno cavernoso agudo, condición caracterizada por la parálisis de diversos nervios craneales. Esta se manifiesta con oftalmoplejía, ptosis y pérdida sensorial facial debido a la afectación de nervios craneales adyacentes ${ }^{4}$.

Las causas comunes de este síndrome son enfermedades inflamatorias del seno cavernoso, neoplásicas, vasculares e infecciosas ${ }^{23}$. En los casos de presentación aguda, lo más frecuente es que la causa sea inflamatoria (entre ellas, el síndrome de Tolosa-Hunt) o una fístula carotidocavernosa. El mejor método para el diagnóstico de estas lesiones es la RM ${ }^{5,23}$.

En este caso, junto a las manifestaciones del síndrome del seno cavernoso, el paciente presentaba proptosis, edema palpebral, ingurgitación de vasos venosos epiesclerales e hipertensión ocular, lo cual sugirió la presencia de un cuadro de congestión venosa orbitaria. En la RM se encontró una lesión metastásica en el seno cavernoso izquierdo que justificó el cuadro clínico.

En este paciente solo se recoge el antecedente de haber sido operado de hipernefroma. Otros autores han reportado casos con síndrome del seno cavernoso secundario a metástasis de lesiones malignas ${ }^{10,24-28}$.

\section{Conclusiones}

En caso de proptosis aguda dolorosa, dos diagnósticos a tener en cuenta son la trombosis de la vena oftálmica superior y las lesiones metastásicas en el seno cavernoso, sobre todo cuando el paciente tiene el antecedente de una enfermedad maligna previamente diagnosticada. Los estudios imagenológicos, específicamente la RM, desempeñan un papel fundamental, pues ayudan a confirmar el diagnóstico y a excluir otras alteraciones que pueden simular estas condiciones.

\section{Conflicto de intereses}

Los autores declaran no tener ningún conflicto de intereses.

\section{Responsabilidades éticas}

Protección de personas y animales. Los autores declaran que para esta investigación no se han realizado experimentos en seres humanos ni en animales.

Confidencialidad de los datos. Los autores declaran que han seguido los protocolos de su centro de trabajo sobre la publicación de datos de pacientes.

Derecho a la privacidad y consentimiento informado. Los autores han obtenido el consentimiento informado de los pacientes y/o sujetos referidos en el 


\section{artículo. Este documento obra en poder del autor de correspondencia.}

\section{Bibliografía}

1. Bauer J, Kansagra K, Chao KH, Feng L. Transfemoral thrombectomy in the cavernous sinus and superior ophthalmic vein. J Neurointerv Surg. 2018;10:e8.

2. Vahdani K, Rose GE. Acute spontaneous rupture of the superior ophthalmic vein. Ophthalmic Plast Reconstr Surg. 2020;36:545-8.

3. Strianese D, Tranfa F, Finelli M, Iuliano A, Staibano S, Mariniello G. Inflammatory myofibroblastic tumor of the orbit: a clinico-pathological study of 25 cases. Saudi J Ophthalmol. 2018;32:33-9.

4. Nambiar R, Nair SG. Cavernous sinus syndrome. Proc (Bayl Univ Med Cent). 2017;30:455-6.

5. Sun HL, Cui SL, Liu L, Jiang HQ, Zhang XJ, Wang JW. Clinical and imaging features and etiologic diagnosis value in patients with cavernous sinus lesion presenting with ophthalmoplegia. Zhonghua Yi Xue Za Zhi. 2018;98:202-7.

6. Amir SP, Kamaruddin MI, Akib MNR, Sirajuddin J. Orbital cellulitis clinically mimicking rhabdomyosarcoma. Int Med Case Rep J. 2019;12:285-9.

7. Topilow NJ, Tran AQ, Koo EB, Alabiad CR. Etiologies of proptosis: a review. Intern Med Rev (Wash D C). 2020;6:10.18103/imr.v6i3.852.

8. Goyal P, Lee S, Gupta N, Kumar Y, Mangla M, Hooda K, et al. Orbital apex disorders: imaging findings and management. Neuroradiol $\mathrm{J}$. 2018;31:104-25

9. Cumurcu T, Demirel S, Keser S, Bulut T, Cavdar M, Do囚an M, et al. Superior ophthalmic vein thrombosis developed after orbital cellulitis. Semin Ophthalmol. 2013;28:58-60.

10. Yang WJ, Zhou YY, Zhao F, Mei ZM, Li S, Xiang Y. Orbital neuroblastoma metastasis. Medicine. 2019;98:36(e17038)

11. Yuan WH, Lin TC, Lirng JF, Guo WY, Chang FP, Ho DM. Computed tomography and magnetic resonance imaging findings of intraorbital granular cell tumor (Abrikossoff's tumor): a case report. J Med Case Rep. 2016;10:119.

12. Mandi区 JJ, Mandi区 K, Mrazovac D. Superior ophthalmic vein thrombosis with complete loss of vision as a complication of autoimmune and infective conditions. Ocul Immunol Inflamm. 2017;24:1-3.

13. Sotoudeh H, Shafaat O, Aboueldahab N, Vaphiades M, Sotoudeh E, Bernstock J. Superior ophthalmic vein thrombosis: What radiologist and clinician must know? Eur J Radiol Open. 2019;6:258-64.
14. Rao R, Ali Y, Nagesh CP, Nair U. Unilateral isolated superior ophthalmic vein thrombosis. Indian J Ophthalmol. 2018;66:155-7.

15. Mishima M, Yumoto T, Hashimoto H, Yasuhara T, Lida T, Tsukahara K, et al. Superior ophthalmic vein thrombosis associated with severe facial trauma: a case report. J Med Case Rep. 2015;9:244

16. Syed A, Bell B, Hise J, Philip J, Spak C, Opatowsky MJ. Bilateral cavernous sinus and superior ophthalmic vein thrombosis in the setting of facial cellulitis. Proc (Bayl Univ Med Cent). 2016;29:36-8.

17. van der Poel NA, de Witt KD, van den Berg R, de Win MM, Mourits MP. Impact of superior ophthalmic vein thrombosis: a case series and literature review. Orbit. 2019;38:226-32.

18. Habib N, Lessard K. Superior and inferior ophthalmic vein thrombosis in the setting of lung cancer. Case Rep Oncol Med. 2018;2018:6025274.

19. Baidoun $F$, Issa R, Ali R, Al-Turk B. Acute unilateral blindness from superior ophthalmic vein thrombosis: a rare presentation of nephrotic syndrome from class iv lupus nephritis in the absence of antiphospholipid or anticardiolipin syndrome. Case Rep Hematol. 2015;2015:413975.

20. Sambhav K, Shakir O, Chalam KV. Bilateral isolated concurrent superior ophthalmic vein thrombosis in systemic lupus erythematosus. Int Med Case Rep J. 2015;8:181-3.

21. Singh K, Gaindh D, Mustafa G, Kamal H, Mowla A. Spontaneous superior ophthalmic vein thrombosis: a case report. Neurology. 2015;84:227.

22. Lim LH, Scawn RL, Whipple KM, Oh SR, Lucarelli MJ, Korn BS, et al. Spontaneous superior ophthalmic vein thrombosis: a rare entity with potentially devastating consequences. Eye (Lond). 2014;28:348-51.

23. Weerasinghe D, Lueck CJ. Septic cavernous sinus thrombosis: case report and review of the literature. Neuroophthalmology. 2016;40:263-76.

24. Mendelson ZS, Patel AA, Eloy JA, Liu JK. Endoscopic palliative descompression of the cavernous sinus in a rare case of a metastatic renal cell carcinoma to the clivus. Br Neurosurg. 2015;29:430-1.

25. Asano Y, Takahashi K, Tsuda H, Takagi M, Kodaka F, Yamada Y. Isolated oculomotor nerve palsy due to metastasis of prostatic cancer to the cavernous sinus. Neuroophthalmology. 2016;40:90-2.

26. Machío Castelló M, Escobar Montatixe D, Cenjor Español C, Villacampa Aubá JM, Montoya Bordón J, Carias Calix R, et al. Incomplete cavernous sinus syndrome as the initial manifestation of a previously undetected metastatic prostate adenocarcinoma. Head Neck. 2017;39:E77-80.

27. Rakul Nambiara K. Ajithb PS, Arjunanc A. Unilateral proptosis as the initial manifestation of malignancy. J Egypt Natl Canc Inst. 2017:29:159-61.

28. Nassrallah G, Sun V, Guiot MC, Mikhail M, Arthurs B. Cavernous sinus syndrome associated with metastatic colorectal cancer and perineural spread along the trigeminal nerve. Am J Ophthalmol Case Rep. 2017;6:67-70. 